Pacific

Journal of

Mathematics

\title{
NO HYPERBOLIC PANTS \\ FOR THE 4-BODY PROBLEM WITH STRONG POTENTIAL
}

CONNOR JACKMAN AND RICHARD MONTGOMERY 


\title{
NO HYPERBOLIC PANTS FOR THE 4-BODY PROBLEM WITH STRONG POTENTIAL
}

\author{
CONNOR JACKMAN AND RICHARD MONTGOMERY
}

\begin{abstract}
The $N$-body problem with a $1 / r^{2}$ potential has, in addition to translation and rotational symmetry, an effective scale symmetry which allows its zero energy flow to be reduced to a geodesic flow on complex projective $(N-2)$ space, minus a hyperplane arrangement. When $N=3$ we get a geodesic flow on the 2-sphere minus three points. If, in addition we assume that the three masses are equal, then it was proved in a previous paper that the corresponding metric is hyperbolic: its Gaussian curvature is negative except at two points. Does the negative curvature property persist for $N=4$, that is, in the equal mass $1 / r^{2}$ potential 4-body problem? Here we prove that it does not by computing that the corresponding Riemannian metric in this $N=4$ case has positive sectional curvature at some 2-planes. This curvature computation underlines an essential difference between the 3- and 4-body problem, a difference whose consequences remain to be explored.
\end{abstract}

\section{Introduction}

In [Montgomery 2005] it was shown that the reduced Jacobi-Maupertuis metric for a certain 3-body problem had negative Gaussian curvature (except at two points where it is zero). This hyperbolicity led to deep dynamical consequences. Does hyperbolicity, i.e., curvature negativity, persist for the analogous $N$-body problem with $N>3$ ? No. We show that the analogous reduced 4-body problem with its metric has 2-planes at which the sectional curvature is positive.

The $N$-body problem in question has equal masses and the inverse cube law attractive force between bodies.

\section{Setup}

Identify the complex numbers $\mathbb{C}$ with the Euclidean plane $\mathbb{R}^{2}$. Then the planar $N$-body problem has configuration space $\mathbb{C}^{N} \backslash \Delta$. Here $\Delta$ is the "fat diagonal"

MSC2010: 37N05, 70F10, 70G45.

Keywords: $N$-body problems, dynamical systems, differential geometry, hyperbolic flows. 
consisting of all collisions:

$$
\Delta=\left\{q=\left(q_{1}, q_{2}, \ldots, q_{N}\right) \in \mathbb{C}^{N}: q_{i}=q_{j} \text { for some pair } i \neq j\right\} .
$$

The quotient of $\mathbb{C}^{N} \backslash \Delta$ by translations and rotations is the "reduced $N$-body configuration space":

$$
C_{N}=Y_{N} \times \mathbb{R}^{+}, \quad Y_{N}=\mathbb{C} \mathbb{P}^{N-2} \backslash \mathbb{P} \Delta,
$$

where $\mathbb{C P} \mathbb{P}^{N-2}$ is the projectivization of the center of mass subspace

$$
\mathbb{C}^{N-1}=\left\{q \in \mathbb{C}^{N}: \sum m_{i} q_{i}=0\right\}
$$

and $\mathbb{P} \Delta \subset \mathbb{C P} \mathbb{P}^{N-2}$ is the projectivization of $\Delta \cap \mathbb{C}^{N-1}$. The $\mathbb{R}^{+}$factor records the overall scale of the planar $N$-gon and is coordinatized by $\sqrt{I}$ with $I=\Sigma m_{i}\left|q_{i}\right|^{2}$ being the total moment of inertia about the center of mass. $Y_{N}$ is the moduli space of oriented similarity classes of noncollision $N$-gons and will be called "shape space".

The following considerations reduce the zero angular momentum, zero energy $N$-body problem to a geodesic flow on shape space $Y_{N}$, provided the potential $V$ is homogeneous of degree -2 . If $V$ is homogeneous of degree $-\alpha$ then the virial identity, also known as the Lagrange-Jacobi identity, asserts that along solutions of energy $H$ we have $\ddot{I}=4 H-(4-2 \alpha) V$, which implies that the only case in which we can generally guarantee that $\ddot{I}=0$ is when $\alpha=2$ and $H=0$. If in addition $\dot{I}=0$ then solutions lie on constant levels of $I$.

Now we recall the Jacobi-Maupertuis (JM) reformulation of mechanics, which asserts that the solutions to Newton's equations at energy $H$ are, after a time reparametrization, precisely the geodesic equations for the Jacobi-Maupertuis metric

$$
d s_{\mathrm{JM}}^{2}=2(H-V) d s^{2}
$$

on the Hill region $\{H-V \geq 0\} \subset \mathbb{C}^{N} \backslash \Delta$ with $d s^{2}$ the mass metric. We are interested in the case $H=0,-V>0$ with $V$ homogeneous of degree -2 , in which case the Hill region is all of $\mathbb{C}^{N} \backslash \Delta$ and

$$
d s_{\mathrm{JM}}^{2}=U d s^{2}, \quad U=-V .
$$

The case of prime interest to us is

$$
U=-V=\sum_{i \neq j} m_{i} m_{j} / r_{i j}^{2} .
$$

This $U$, and hence the JM metric, is invariant under rotations and translations. Quotienting first by translations, we take representatives in the totally geodesic center-of-mass-zero subspace $\mathbb{C}^{N-1}$, which reduces the dynamics to geodesics of the metric $\left.d s_{\mathrm{JM}}^{2}\right|_{\mathbb{C}^{N-1}}$ on $\mathbb{C}^{N-1}$. Moreover, $\left.d s_{\mathrm{JM}}^{2}\right|_{\mathbb{C}^{N-1}}$ is also invariant under scaling since 
the homogeneities of $U$ and the Euclidean mass metric $d s^{2}$ on $\mathbb{C}^{N-1}$ cancel. Thus the JM metric admits the group $G=\mathbb{C}^{*}$ of rotations and scalings as an isometry group.

Now $Y_{N}$ is the quotient space: $Y_{N}=\left(\mathbb{C}^{N-1} \backslash \Delta\right) / G=\mathbb{C P} \mathbb{P}^{N-2} \backslash \Delta$. (By abuse of notation, we continue to use the symbol $\Delta$ to denote the image of the collision locus $\Delta$ under projectivization and intersection.) Insisting that the quotient map $\pi: \mathbb{C}^{N-1} \backslash \Delta \rightarrow Y_{N}$ is a Riemannian submersion induces a metric on $Y_{N}$. Recall that this means that we can define the metric on $Y_{N}$ by isometrically identifying the tangent space to $Y_{N}$ at a point $p$ with the orthogonal complement (relative to $d s_{\mathrm{JM}}^{2}$ or $d s^{2}$, and at any point lying over $p$ in $\mathbb{C}^{N-1}$ ) to the $G$-orbit that corresponds to that point. These orthogonality conditions are equivalent to the conditions that the linear momentum, angular momentum, and "scale momentum" $\dot{I}$ are all zero. To summarize, by using the JM metric and forming the Riemannian quotient, the zero angular momentum, zero energy $1 / r^{2}$ potential $N$-body problem becomes equivalent to the problem of finding geodesics for the metric defined by Riemannian submersion on $Y_{N}$.

Remark. The metric quotient procedure just described realizes the MarsdenWeinstein symplectic reduced space of $T^{*}\left(\mathbb{C}^{N} \backslash \Delta\right)$ by the action of translations, rotations and scalings, $\mathbb{C} \rtimes \mathbb{C}^{*}$, at momentum values 0 , together with the $N$-body reduced Hamiltonian flow, but valid only at zero energy.

Remark. This metric on $Y_{N}$ can be expressed as $U d s_{\mathrm{FS}}^{2}$ where $d s_{\mathrm{FS}}^{2}$ is the usual Fubini-Study metric on $\mathbb{C} \mathbb{P}^{N-2}$.

Remark. For the standard $1 / r^{2}$ potential of (1), this metric on $Y_{N}$ is complete, with infinite volume.

The collinear $N$-body problem defines a totally geodesic submanifold

$$
\mathbb{R P}^{N-2} \backslash \Delta \subset \mathbb{C P}^{N-2} \backslash \Delta \text {. }
$$

We obtain this submanifold by placing the $N$-masses anywhere along the real axis $\mathbb{R} \subset \mathbb{C}$, arranged so their center of mass is zero and so that there are no collisions, and then taking the quotient. In other words, $\mathbb{R} \mathbb{P}^{N-2} \backslash \Delta$ is the quotient of $\mathbb{R}^{N-1} \subset \mathbb{C}^{N-1}$ by dilations and real reflections.

\section{Main result}

In case $N=3$, with the potential (1) above, $Y_{3}$ is a pair of pants - a sphere minus three points. The point of [Montgomery 2005] was to show that the metric on $Y_{3}$ just described is hyperbolic provided $m_{1}=m_{2}=m_{3}$. Specifically, in this equal mass case the Gaussian curvature of the metric on the surface $Y_{3}$ is negative everywhere except at two points (these being the "Lagrange points" corresponding to equilateral triangles.) What about $Y_{4}$ ? 


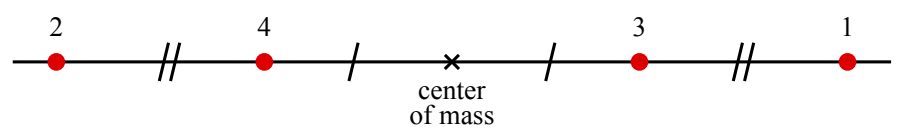

Figure 1. The collinear configurations $p$ which we consider.

Theorem 1. Consider the Jacobi-Maupertuis metric on $Y_{4}$ induced as above for the case of 4 equal masses under the strong force $1 / r^{2}$ potential (1). Then there are 2-planes $\sigma$ tangent to $Y_{4}$ at which the Riemannian sectional curvature $\mathcal{K}(\sigma)$ is positive.

Remark. The 2-planes $\sigma$ of the theorem pass through special points $p \in \mathbb{R} \mathbb{P}^{2} \subset \mathbb{C P}^{2}$ which represent certain special collinear configurations. See Figure 1. The 2-plane $\sigma$ at $p$ will be the orthogonal complement to $T_{p} \mathbb{R P}^{2}$, the normal 2-plane, and is realized as $\sigma=i T_{p} \mathbb{R} \mathbb{P}^{2}$, using the standard complex structure on $\mathbb{C} \mathbb{P}^{2}$.

Remark (negative curvatures). The $\mathbb{R} \mathbb{P}^{2}$ of the previous remark is a totally geodesic surface fixed by an isometric involution. There are other such totally geodesic surfaces defined as fixed loci of symmetries, and computer experiments suggest that these all have negative Gaussian curvature everywhere while their normal 2-planes can have positive sectional curvature at some points, like our special case $\mathbb{R} \mathbb{P}^{2}$. Computer experiments also indicate that in the direction of the normal plane there is positive sectional curvature over all collinear configurations of $\mathbb{R P}^{2}$ and not just the special configurations verified in the theorem. An analytic proof of these claims beyond our special case, however, looks frightening.

Remark (uniqueness of free homotopy classes). The work in [Montgomery 2005] was chiefly meant as a route for proving the uniqueness (mod symmetries) of the $N=3$ strong force figure-eight solution. For $N=4$, hyperbolicity fails and we have no direct "hyperbolic" path for establishing uniqueness of various 4-body choreographies or free homotopy class representatives.

Open Question. A geodesic flow can still be hyperbolic as a flow, without the underlying metric having all sectional curvatures negative. Is geodesic flow on $Y_{4}$ hyperbolic as a flow? Is it even partially hyperbolic?

\section{Proof of the theorem}

We take the case $N=4$ in the above considerations. When all the masses are equal to 1 , the mass metric, used to compute the kinetic energy and moment of inertia, is the standard Hermitian metric in coordinates $\left(q_{1}, q_{2}, q_{3}, q_{4}\right) \in \mathbb{C}^{4}$, where the $q_{i}$ represent the positions of the $i$-th body. We reduce by translations by going to the center-of-mass-zero space, which is a 3-dimensional subspace $\mathbb{C}^{3} \subset \mathbb{C}^{4}$ having 
Jacobi coordinates as Hermitian orthonormal coordinates:

$\mathbb{C}^{3} \stackrel{L}{\rightarrow} \mathbb{C}^{4}$ given by the matrix $\left[\begin{array}{rrr}\frac{1}{2} & \frac{1}{\sqrt{2}} & 0 \\ \frac{1}{2} & -\frac{1}{\sqrt{2}} & 0 \\ -\frac{1}{2} & 0 & \frac{1}{\sqrt{2}} \\ -\frac{1}{2} & 0 & -\frac{1}{\sqrt{2}}\end{array}\right]$ in standard bases.

As is well-known, if we start tangent to the center-of-mass-zero subspace $L\left(\mathbb{C}^{3}\right)$, we stay tangent to it. Hence we can restrict the dynamics, potential, metric, etc. to the center-of-mass-zero subspace. We denote the potential restricted to the center-of-mass-zero subspace in Jacobi coordinates as $U_{L}=U \circ L$ and still write $d s_{\mathrm{JM}}^{2}=U_{L} d s^{2}$ for the restricted JM metric on $\mathbb{C}^{3} \backslash \Delta$ where $d s^{2}$ is the standard metric on $\mathbb{C}^{3}$.

Continuing along the outline above, we now quotient by scaling and rotation isometries $\mathbb{C}^{*}$ of $d s_{\mathrm{JM}}^{2}$ to obtain the "shape space" $Y_{4}$ and we label the quotient map $\pi: \mathbb{C}^{3} \backslash \Delta \rightarrow Y_{4}$, which takes a configuration $q$ to its orbit $\mathbb{C}^{*} q$. We denote the vertical and horizontal distributions as

$$
\mathcal{V}_{p}=\operatorname{ker} d_{p} \pi=\mathbb{C} p \quad \text { and } \quad \mathcal{H}_{p}=\mathcal{V}_{p}^{\perp} \stackrel{d \pi}{\cong} T_{\pi(p)} Y_{4} .
$$

Requiring $\left.d \pi\right|_{\mathcal{H},\left.d s_{\mathrm{JM}}^{2}\right|_{\mathcal{H}}}$ to be an isometry defines our induced metric on $Y_{4}$ whose geodesics correspond to $N$-body motions in "shape space". Under this induced metric on $Y_{4}$ we denote sectional curvature through the plane $\sigma \in T_{\pi(p)} Y_{4}$ by $\mathcal{K}(\sigma)$.

Suppressing the notation of evaluating at a representative $p \in \pi(q)$, our main tool in the computation of $\mathcal{K}(\sigma)$, the $d s_{\mathrm{JM}}^{2}$ curvature, is the equation

$$
\begin{aligned}
& U_{L}^{3} \mathcal{K}(\sigma) \\
& =\frac{3}{4}\left(\left(\partial_{1} U_{L}\right)^{2}+\left(\partial_{2} U_{L}\right)^{2}\right)-\left\|\frac{\nabla U}{2}\right\|^{2}-\frac{U_{L}}{2}\left(\partial_{1}^{2} U_{L}+\partial_{2}^{2} U_{L}\right)+3 \frac{U_{L}^{2}}{\|p\|^{2}}\left(v_{1} \cdot i v_{2}\right)^{2}
\end{aligned}
$$

Here $\partial_{a} f$ denotes $d f\left(v_{a}\right)$ where $f \in C^{\infty}\left(\mathbb{C}^{3}\right)$ and where $a=1,2$ with $v_{1}, v_{2} \in \mathcal{H}$ being $d s^{2}$-orthonormal vectors whose pushforwards $d \pi v_{a}$ span $\sigma$. The $\cdot,\|\|$, and $\nabla$ refer to the norm, metric, and Levi-Civita connection for the Euclidean metric $d s^{2}$. For the derivation of (2) see the Appendix.

The collinear configurations form a totally geodesic projective plane $\mathbb{R} \mathbb{P}^{2} \subset \mathbb{C} \mathbb{P}^{2}$, the image under $\pi$ of the real 2-sphere in $\mathbb{C}^{3}$, which we parametrize by

$$
p=(\cos \phi \cos \theta, \cos \phi \sin \theta, \sin \phi) .
$$

We evaluate (2) and find positive sectional curvature over the configurations with $\theta=\pi / 2$ (see Figure 1) in the direction of the $i T \mathbb{R} \mathbb{P}^{2}$ plane. This plane is spanned 
by the pushforwards of

$$
\begin{aligned}
& v_{1}=-i \frac{\partial p}{\partial \phi}=i(\sin \phi \cos \theta, \sin \phi \sin \theta,-\cos \phi), \\
& v_{2}=\frac{i}{\cos \phi} \frac{\partial p}{\partial \theta}=i(-\sin \theta, \cos \theta, 0) .
\end{aligned}
$$

Terms 1. Over $\mathbb{R P}^{2}$ in the $i T \mathbb{R} \mathbb{P}^{2}$ direction, the last and first summands on the second line of (2) vanish:

$$
v_{1} \cdot i v_{2}=0, \quad \partial_{a} U_{L}=0 .
$$

Proof. That $v_{1} \cdot i v_{2}=0$ is clear: $i$ rotates $v_{2}$ into purely real coordinates. To evaluate the first partials, note that $L p$ has purely real coordinates and $\nabla U$ has $k$-th component $\sum_{j \neq k}\left(q_{j}-q_{k}\right) / r_{j k}^{4}$, so $\left.\nabla\right|_{L p} U$ has purely real coordinates. Now since $L v_{a}$ has purely complex coordinates,

$$
\partial_{a} U_{L}=\left.\nabla\right|_{L p} U \cdot L v_{a}=0 .
$$

Terms 2. With the notation $L p=\left(q_{1}, q_{2}, q_{3}, q_{4}\right), L v_{a}=i\left(v_{a}^{1}, v_{a}^{2}, v_{a}^{3}, v_{a}^{4}\right)$, and

$$
\rho_{j k}=1 /\left(q_{j}-q_{k}\right), \alpha_{j k}=\left(v_{1}^{j}-v_{1}^{k}\right)^{2}+\left(v_{2}^{j}-v_{2}^{k}\right)^{2} \in \mathbb{R},
$$

the sum of second partials in (2) is given by

$$
\partial_{1}^{2} U_{L}+\partial_{2}^{2} U_{L}=-2 \sum_{j>k} \alpha_{j k} \rho_{j k}^{4}
$$

Proof. We write our standard coordinates on $\mathbb{C}^{4}$ as $q_{j}=x_{j}+i y_{j}$. Then since $L v_{a}$ is purely imaginary, we have

$$
\partial_{a}^{2} U_{L}=\left.\nabla\right|_{L p}\left(\nabla U \cdot L v_{a}\right) \cdot L v_{a}=\left(\left.\nabla\right|_{L p} \frac{\partial U}{\partial y_{k}} v_{a}^{k}\right) \cdot L v_{a}=\left.\frac{\partial^{2} U}{\partial y_{j} \partial y_{k}}\right|_{L p} v_{a}^{k} v_{a}^{j} .
$$

Next we compute $\left.\frac{\partial^{2} U}{\partial y_{j} \partial y_{k}}\right|_{L p}=2 \rho_{j k}^{4}$ for $j \neq k$ and $\left.\frac{\partial^{2} U}{\partial y_{k}^{2}}\right|_{L p}=-2 \sum_{j \neq k} \rho_{j k}^{4}$, so now

$$
\begin{aligned}
\partial_{a}^{2} U_{L} & =-2 \sum_{j \neq k} \rho_{j k}^{4}\left(\left(v_{a}^{k}\right)^{2}-v_{a}^{j} v_{a}^{k}\right) \\
& =-2 \sum_{j>k} \rho_{j k}^{4}\left(\left(v_{a}^{k}\right)^{2}-2 v_{a}^{k} v_{a}^{j}+\left(v_{a}^{j}\right)^{2}\right) \\
& =-2 \sum_{j>k} \rho_{j k}^{4}\left(v_{a}^{k}-v_{a}^{j}\right)^{2} .
\end{aligned}
$$

Result. Over the circle $\theta=\pi / 2, \mathcal{K}\left(i T \mathbb{R} \mathbb{P}^{2}\right)$ is positive. 
Now, substituting Terms 1 and 2 into formula (2), we see that

$$
\begin{aligned}
0<\mathcal{K} & \Longleftrightarrow 0<U_{L}^{3} \mathcal{K}=-\|\nabla U / 2\|^{2}+U_{L} \sum_{j>k} \alpha_{j k} \rho_{j k}^{4} \\
& \Longleftrightarrow \sum_{k}\left(\sum_{j \neq k} \rho_{j k}^{3}\right)^{2}<\left(\sum_{j>k} \rho_{j k}^{2}\right)\left(\sum_{j>k} \alpha_{j k} \rho_{j k}^{4}\right) .
\end{aligned}
$$

Taking $\theta=\pi / 2$ and with the notation introduced in Terms 2 , we find the relations

$$
\begin{array}{cc}
\rho_{12}=\frac{1}{\sqrt{2} \cos \phi}, \quad \rho_{34}=\frac{1}{\sqrt{2} \sin \phi} & \alpha_{12}=\frac{1}{\rho_{34}^{2}}, \quad \alpha_{34}=\frac{1}{\rho_{12}^{2}} \\
\rho_{13}=\frac{\sqrt{2}}{\cos \phi-\sin \phi}=-\rho_{24} & \alpha_{13}=\frac{1}{\rho_{14}^{2}}+1=\alpha_{24} \\
\rho_{14}=\frac{\sqrt{2}}{\cos \phi+\sin \phi}=-\rho_{23} & \alpha_{14}=\frac{1}{\rho_{13}^{2}}+1=\alpha_{23} .
\end{array}
$$

Now the left side of (3) works out to

$$
\begin{aligned}
2\left(\left(\rho_{12}^{3}+\rho_{13}^{3}+\rho_{14}^{3}\right)^{2}\right. & \left.+\left(\rho_{13}^{3}-\rho_{14}^{3}-\rho_{34}^{3}\right)^{2}\right) \\
& =2\left(\sum_{k>j} \rho_{j k}^{6}+2 \rho_{12}^{3}\left(\rho_{13}^{3}+\rho_{14}^{3}\right)+2 \rho_{34}^{3}\left(\rho_{14}^{3}-\rho_{13}^{3}\right)\right) \\
& =2 \sum_{k>j} \rho_{j k}^{6}-96 \frac{1}{\sin ^{2} 2 \phi \cos ^{2} 2 \phi}=2 \sum_{k>j} \rho_{j k}^{6}+\text { negative term, }
\end{aligned}
$$

and the right side of (3) works out to

$$
\begin{aligned}
\left(\rho_{12}^{2}+\rho_{34}^{2}+\right. & \left.2\left(\rho_{13}^{2}+\rho_{14}^{2}\right)\right)\left(\frac{\rho_{12}^{4}}{\rho_{34}^{2}}+\frac{\rho_{34}^{4}}{\rho_{12}^{2}}+2\left(\rho_{13}^{4}+\rho_{14}^{4}+\frac{\rho_{13}^{4}}{\rho_{14}^{2}}+\frac{\rho_{14}^{4}}{\rho_{13}^{2}}\right)\right) \\
= & \left(\frac{2}{\sin ^{2} 2 \phi}+\frac{8}{\cos ^{2} 2 \phi}\right)\left(\sin ^{2} 2 \phi\left(\rho_{12}^{6}+\rho_{34}^{6}\right)\right. \\
& \left.\quad+\frac{\cos ^{2} 2 \phi}{2}\left(\rho_{13}^{6}+\rho_{14}^{6}\right)+2\left(\rho_{13}^{4}+\rho_{14}^{4}\right)\right) \\
= & 2 \sum_{k>j} \rho_{j k}^{6}+\cot ^{2} 2 \phi\left(\rho_{13}^{6}+\rho_{14}^{6}\right)+8 \tan ^{2} 2 \phi\left(\rho_{12}^{6}+\rho_{34}^{6}\right) \\
& +\left(\rho_{13}^{4}+\rho_{14}^{4}\right)\left(\frac{4}{\sin ^{2} 2 \phi}+\frac{16}{\cos ^{2} 2 \phi}\right) \\
= & 2 \sum_{k>j} \rho_{j k}^{6}+\text { positive term. }
\end{aligned}
$$

Therefore the inequality (3) holds! 


\section{Appendix: Derivation of (2)}

Take a $d s^{2}$-orthonormal basis $\left\{v_{a}\right\}$ for $\mathbb{C}^{3}$ with $v_{1}, v_{2} \in \mathcal{H}_{p}$.

The Kulkarni-Nomizu product formula for conformal curvatures [Sakai 1996, p. 51] reads:

$$
\bar{R}_{a b c d}-U_{L} R_{a b c d}=-\left\{d s_{\mathrm{JM}}^{2} \otimes\left(\nabla d u-d u \otimes d u+\frac{1}{2}\|d u\|^{2} d s^{2}\right)\right\}_{a b c d}
$$

where $u:=\frac{1}{2} \log U_{L}$, the overbars denote curvature with respect to the $d s_{\mathrm{JM}}^{2}$-metric, and all other quantities (without overbars) are with respect to the $d s^{2}$-metric. Then $R_{a b c d}=0$ since $d s^{2}$ is the flat Euclidean metric of $\mathbb{C}^{3}=\mathbb{R}^{6}$. Taking $c d=a b$, we have

$$
\begin{aligned}
U_{L}^{2} \bar{K}_{a b}=\bar{R}_{a b a b} & =-U_{L}\left(\nabla d u_{b b}+\nabla d u_{a a}-d u_{b} \otimes d u_{b}-d u_{a} \otimes d u_{a}+\|d u\|^{2}\right) \\
& =-U_{L}\left(\partial_{a}^{2} u+\partial_{b}^{2} u-\left(\partial_{a} u\right)^{2}-\left(\partial_{b} u\right)^{2}+\|\nabla u\|^{2}\right) .
\end{aligned}
$$

Next, O’Neill's formula [1983, p. 213] gives

$$
\mathcal{K}\left(d \pi v_{1}, d \pi v_{2}\right)=\bar{K}_{12}+\frac{3}{4}\left|\left[V_{1}, V_{2}\right]^{\mathcal{V}}\right|_{d s_{\mathrm{JM}}^{2}}^{2},
$$

where $V_{a}=v_{a} / \sqrt{U_{L}(p)}$ and $X^{\mathcal{V}}$ denotes $d s_{\mathrm{JM}}^{2}$ projection of $X$ onto $\mathcal{V}$.

We then compute

$$
\partial_{a} u=\frac{\partial_{a} U_{L}}{2 U_{L}}=\frac{\left.\nabla\right|_{L p} U \cdot L v_{a}}{2 U_{L}(p)}
$$

and

$$
\begin{aligned}
\partial_{a}^{2} u & =\frac{\partial_{a}^{2} U_{L}}{2 U_{L}}-\frac{\left(\partial_{a} U_{L}\right)^{2}}{2 U_{L}^{2}} \\
& =\frac{\left.\nabla\right|_{L p}\left(\nabla U \cdot L v_{a}\right) \cdot L v_{a}}{2 U_{L}(p)}-\frac{\left(\partial_{a} U_{L}\right)^{2}}{2 U_{L}(p)^{2}} .
\end{aligned}
$$

Note that $\nabla U \in\left\{q \in \mathbb{C}^{4}: \sum q_{j}=0\right\}$ and $L v_{a}$ is a $d s^{2}$ orthonormal basis for this center-of-mass-zero subspace, hence

$$
\begin{aligned}
\|\nabla U\|^{2} & =\sum\left(\nabla U \cdot L v_{a}\right)^{2} \\
& =\sum\left(\partial_{a} U_{L}\right)^{2}=4 U_{L}^{2}\|\nabla u\|^{2} .
\end{aligned}
$$

Substitution into the Kulkarni-Nomizu formula gives

$$
\bar{K}_{12}=-\frac{1}{U_{L}^{3}}\left(\frac{U_{L}}{2}\left(\partial_{1}^{2} U_{L}+\partial_{2}^{2} U_{L}\right)-\frac{3}{4}\left(\partial_{1} U_{L}^{2}+\partial_{2} U_{L}^{2}\right)+\left\|\frac{\nabla U}{2}\right\|^{2}\right) .
$$

To compute O'Neill's Lie bracket term we write our standard coordinates on $\mathbb{C}^{3}$ as $\left(x^{1}+i x^{2}, \ldots, x^{5}+i x^{6}\right)$. 
Let $H_{1}=X^{j} \partial_{x^{j}}, H_{2}=Y^{j} \partial_{x^{j}} \in \mathcal{H}$ be any horizontal vector fields. The vertical vector fields are spanned by the Euler vector field $E=x^{j} \partial_{x^{j}}$ and $i E$. Then $H_{j} \cdot E=H_{j} \cdot i E=0$ and

$$
\begin{aligned}
{\left[H_{1}, H_{2}\right] \cdot E } & =\sum_{k} X^{j} x^{k} \partial_{x^{j}} Y^{k}-Y^{j} x^{k} \partial_{x^{j}} X^{k} \\
& =\sum_{k} X^{j}\left(\partial_{x^{j}}\left(x^{k} Y^{k}\right)-\delta_{j}^{k} Y^{k}\right)-Y^{j}\left(\partial_{x^{j}}\left(x^{k} X^{k}\right)-\delta_{j}^{k} X^{k}\right) \\
& =\sum_{k} X^{k} Y^{k}-Y^{k} X^{k}=0,
\end{aligned}
$$

and likewise,

$$
\begin{aligned}
{\left[H_{1}, H_{2}\right] \cdot i E } & =\sum_{k \text { odd }}\left(Y^{j} \partial_{x^{j}} X^{k}-X^{j} \partial_{x^{j}} Y^{k}\right) x^{k+1}+\left(X^{j} \partial_{x^{j}} Y^{k+1}-Y^{j} \partial_{x^{j}} X^{k+1}\right) x^{k} \\
& =2 \sum_{k \text { odd }}-X^{k} Y^{k+1}+X^{k+1} Y^{k}=2 H_{1} \cdot i H_{2} .
\end{aligned}
$$

Then

$$
\begin{aligned}
\left|\left[V_{1}, V_{2}\right]^{\mathcal{V}_{p}}\right|^{2} & =d s_{\mathrm{JM}}^{2}\left(\left[V_{1}, V_{2}\right], \frac{E_{p}}{|p| \sqrt{U_{L}(p)}}\right)^{2}+d s_{\mathrm{JM}}^{2}\left(\left[V_{1}, V_{2}\right], \frac{i E_{p}}{|p| \sqrt{U_{L}(p)}}\right)^{2} \\
& =\frac{U_{L}^{2}}{|p|^{2} U_{L}}\left(\left(\left[V_{1}, V_{2}\right] \cdot E\right)^{2}+\left(\left[V_{1}, V_{2}\right] \cdot i E\right)^{2}\right) \\
& =\frac{4 U_{L}(p)\left(V_{1} \cdot i V_{2}\right)^{2}}{|p|^{2}}=\frac{4}{U_{L}(p)|p|^{2}}\left(v_{1} \cdot i v_{2}\right)^{2} .
\end{aligned}
$$

Now substitution of this Lie bracket expression and (4) into O'Neill's formula and multiplying by $U_{L}^{3}$ yields (2).

\section{Acknowledgments}

We would like to thank Slobodan Simic of San Jose State University for discussions on hyperbolicity, and Prof. Jie Qing and Wei Yuan of UC Santa Cruz for conversations regarding curvature computations. We thankfully acknowledge support by NSF grant DMS-1305844.

\section{References}

[Montgomery 2005] R. Montgomery, "Fitting hyperbolic pants to a three-body problem", Ergodic Theory Dynam. Systems 25:3 (2005), 921-947. MR 2006b:70013 Zbl 1069.70006

[O’Neill 1983] B. O'Neill, Semi-Riemannian geometry: With applications to relativity, 1st ed., Pure and Applied Mathematics 103, Academic Press, New York, 1983. MR 85f:53002 Zbl 0531.53051

[Sakai 1996] T. Sakai, Riemannian geometry, 1st ed., Translations of Mathematical Monographs 149, Amer. Math. Soc., Providence, RI, 1996. Zbl 0886.53002 
Received February 5, 2015. Revised May 8, 2015.

CONNOR JACKMAN

DEPARTMENT OF MATHEMATICS

UNIVERSITY OF CALIFORNIA

SANTA CRUZ, CA 95064

UNITED STATES

cfjackma@ucsc.edu

RICHARD MONTGOMERY

DEPARTMENT OF MATHEMATICS

UNIVERSITY OF CALIFORNIA

SANTA CRUZ, CA 95064

UNITED STATES

rmont@ucsc.edu 


\title{
PACIFIC JOURNAL OF MATHEMATICS
}

\author{
msp.org/pjm
}

Founded in 1951 by E. F. Beckenbach (1906-1982) and F. Wolf (1904-1989)

\section{EDITORS}

Don Blasius (Managing Editor)

Department of Mathematics

University of California

Los Angeles, CA 90095-1555

blasius@math.ucla.edu

\author{
Paul Balmer \\ Department of Mathematics \\ University of California \\ Los Angeles, CA 90095-1555 \\ balmer@math.ucla.edu \\ Robert Finn \\ Department of Mathematics \\ Stanford University \\ Stanford, CA 94305-2125 \\ finn@math.stanford.edu \\ Sorin Popa \\ Department of Mathematics \\ University of California \\ Los Angeles, CA 90095-1555 \\ popa@math.ucla.edu
}

\author{
Vyjayanthi Chari \\ Department of Mathematics \\ University of California \\ Riverside, CA 92521-0135 \\ chari@math.ucr.edu \\ Kefeng Liu \\ Department of Mathematics \\ University of California \\ Los Angeles, CA 90095-1555 \\ liu@math.ucla.edu \\ Jie Qing \\ Department of Mathematics \\ University of California \\ Santa Cruz, CA 95064 \\ qing@ cats.ucsc.edu
}

\section{PRODUCTION}

Silvio Levy, Scientific Editor, production@msp.org

\section{SUPPORTING INSTITUTIONS}

ACADEMIA SINICA, TAIPEI

CALIFORNIA INST. OF TECHNOLOGY

INST. DE MATEMÁTICA PURA E APLICADA

KEIO UNIVERSITY

MATH. SCIENCES RESEARCH INSTITUTE

NEW MEXICO STATE UNIV.

OREGON STATE UNIV.

\author{
STANFORD UNIVERSITY \\ UNIV. OF BRITISH COLUMBIA \\ UNIV. OF CALIFORNIA, BERKELEY \\ UNIV. OF CALIFORNIA, DAVIS \\ UNIV. OF CALIFORNIA, LOS ANGELES \\ UNIV. OF CALIFORNIA, RIVERSIDE \\ UNIV. OF CALIFORNIA, SAN DIEGO \\ UNIV. OF CALIF., SANTA BARBARA
}

\author{
Daryl Cooper \\ Department of Mathematics \\ University of California \\ Santa Barbara, CA 93106-3080 \\ cooper@math.ucsb.edu \\ Jiang-Hua Lu \\ Department of Mathematics \\ The University of Hong Kong \\ Pokfulam Rd., Hong Kong \\ jhlu@maths.hku.hk \\ Paul Yang \\ Department of Mathematics \\ Princeton University \\ Princeton NJ 08544-1000 \\ yang@math.princeton.edu
}

These supporting institutions contribute to the cost of publication of this Journal, but they are not owners or publishers and have no responsibility for its contents or policies.

See inside back cover or msp.org/pjm for submission instructions.

The subscription price for 2016 is US $\$ 440 /$ year for the electronic version, and $\$ 600 /$ year for print and electronic.

Subscriptions, requests for back issues and changes of subscribers address should be sent to Pacific Journal of Mathematics, P.O. Box 4163, Berkeley, CA 94704-0163, U.S.A. The Pacific Journal of Mathematics is indexed by Mathematical Reviews, Zentralblatt MATH, PASCAL CNRS Index, Referativnyi Zhurnal, Current Mathematical Publications and Web of Knowledge (Science Citation Index).

The Pacific Journal of Mathematics (ISSN 0030-8730) at the University of California, c/o Department of Mathematics, 798 Evans Hall \#3840, Berkeley, CA 94720-3840, is published twelve times a year. Periodical rate postage paid at Berkeley, CA 94704, and additional mailing offices. POSTMASTER: send address changes to Pacific Journal of Mathematics, P.O. Box 4163, Berkeley, CA 94704-0163.

PJM peer review and production are managed by EditFLOW ${ }^{\circledR}$ from Mathematical Sciences Publishers.

\section{PUBLISHED BY}

\section{mathematical sciences publishers \\ nonprofit scientific publishing}

http://msp.org/

(C) 2016 Mathematical Sciences Publishers 


\section{PACIFIC JOURNAL OF MATHEMATICS}

Volume $280 \quad$ No. $2 \quad$ February 2016

Topological Molino's theory

JESÚS A. ÁlVAREz LóPEZ and MANUEl F. MoreIRA GALICIA

Equivariant principal bundles and logarithmic connections on toric varieties

INDRANIL BISWAS, ARIJIT DEY and MAINAK PODDAR

On a spectral theorem in paraorthogonality theory

Kenier CASTILlo, RuYmán CRUZ-BARRoso and Francisco

PERDOMO-PÍO

Sigma theory and twisted conjugacy, II: Houghton groups and pure

symmetric automorphism groups

DACIBERG L. GONÇALVES and PARAMESWARAN SANKARAN

The second CR Yamabe invariant

PAK TUNG Ho

No hyperbolic pants for the 4-body problem with strong potential

CONNOR JACKMAN and RICHARD MONTGOMERY

Unions of Lebesgue spaces and $A_{1}$ majorants

Greg Knese, John E. M ${ }^{\mathrm{C}}$ CARThy and Kabe Moen

Complex hyperbolic $(3,3, n)$ triangle groups

JOHN R. PARKER, JiEYAN WANG and BAOHUA XIE

Topological aspects of holomorphic mappings of hyperquadrics from $\mathbb{C}^{2}$ to $\mathbb{C}^{3}$

\section{MICHAEL REITER}

2-Blocks with minimal nonabelian defect groups III

BENJAMIN SAMBALE

Number of singularities of stable maps on surfaces 